\title{
Neutrophil-to-lymphocyte ratios and platelet-to-lymphocyte ratios in juvenile systemic lupus erythematosus: correlation with disease manifestations
}

\author{
Wengen $\mathrm{Li}^{1} \wedge$, Sudong Liu ${ }^{2,3}$, Chao Chen ${ }^{1}$, Yanying Han ${ }^{1}$ \\ ${ }^{1}$ Rheumatology and Immunology Department, Meizhou People's Hospital (Huangtang Hospital), Meizhou, China; ${ }^{2}$ Clinical Core Laboratory, \\ Meizhou People's Hospital (Huangtang Hospital), Meizhou, China; ${ }^{3}$ Guangdong Provincial Key Laboratory of Precision Medicine and Clinical \\ Translational Research of Hakka Population, Meizhou People's Hospital (Huangtang Hospital), Meizhou, China \\ Contributions: (I) Conception and design: W Li; (II) Administrative support: None; (III) Provision of study materials or patients: C Chen, Y Han; \\ (IV) Collection and assembly of data: C Chen, Y Han, S Liu; (V) Data analysis and interpretation: W Li, S Liu; (VI) Manuscript writing: All authors; \\ (VII) Final approval of manuscript: All authors. \\ Correspondence to: Wengen Li. Rheumatology and Immunology Department, Meizhou People's Hospital (Huangtang Hospital), 63 Huangtang Road, \\ Meijiang District, Meizhou 514031, China. Email: ligen0072008@163.com.
}

\begin{abstract}
Background: This retrospective study aimed to investigate the usefulness of neutrophil-to-lymphocyte ratio (NLR) and platelet-to-lymphocyte ratio (PLR) for organ involvement and disease activity in newly diagnosed juvenile systemic lupus erythematosus (jSLE).

Methods: A total of 186 jSLE inpatients were included for analysis. All participants' clinical characteristics and laboratory data were obtained from medical records. The Systemic Lupus Erythematosus Disease Activity Index 2000 (SLEDAI-2K) score scale was used to assess disease activity. Mann-Whitney U test and Kruskal-Wallis test were performed for non-parametric variables between the groups. Spearman rank correlation coefficient was used to analyze correlations between variables.
\end{abstract}

Results: The NLR was significantly higher in participants with serositis as compared those without serositis [2.72 (1.71-5.0) vs. $2.08(1.42-3.15), \mathrm{P}=0.038]$. The PLR was significantly higher in participants manifesting symptoms of cutaneous rash [130.0 (92.6-235.0) vs. 112.0 (49.3-169.0), $\mathrm{P}=0.002$ ], and arthritis [167.0 (106.0-243.0) vs. 106.0 (53.6-176.0), $\mathrm{P}<0.001$ ], as compared to participants without such involvement. The PLR in participants with hematological involvement was significantly lower than in those without such involvement $[111.0$ (53.6-191.0) vs. 138.0 (107.0-185.0), $\mathrm{P}=0.016]$. The PLR in participants with positive anti-Smith (anti-Sm) antibody was significantly higher than that in those with negative anti-Sm antibody [140.0 (91.6-228.0) vs. 114.0 (60.9-176.0), $\mathrm{P}=0.006]$. The NLR showed positive correlations with serositis ( $\mathrm{r}=0.153, \mathrm{P}=0.037)$, complement $\mathrm{C} 3$ and $\mathrm{C} 4(\mathrm{r}=0.152, \mathrm{P}=0.038$ and $\mathrm{r}=0.177, \mathrm{P}=0.016$, respectively). The PLR showed positive correlations with cutaneous rash $(\mathrm{r}=0.227, \mathrm{P}=0.002)$, arthritis $(\mathrm{r}=0.290, \mathrm{P}<0.001)$, anti-Sm antibodies $(r=0.20, P=0.006)$ and erythrocyte sedimentation rate $(E S R, r=0.159, P=0.03)$. Negative correlations were found between PLR and hematological involvement ( $r=-0.177, \mathrm{P}=0.015)$.

Conclusions: Both the NLR and PLR had correlations with serological indicators, and may predict organ involvement in jSLE, particularly cutaneous, arthritis, serositis, and haematological involvement.

Keywords: Systemic lupus erythematosus (SLE); neutrophil-to-lymphocyte ratio; platelet-to-lymphocyte ratio

Submitted Mar 16, 2021. Accepted for publication Sep 09, 2021.

doi: 10.21037/apm-21-1995

View this article at: https://dx.doi.org/10.21037/apm-21-1995

\footnotetext{
^ ORCID: 0000-0001-7920-6316.
} 


\section{Introduction}

Systemic lupus erythematosus (SLE) is a chronic systemic inflammatory autoimmune disease, characterized by various autoantibodies in serum and involvement of multiple systems. The age of onset of SLE influences its clinical characteristics $(1,2)$. The onset of SLE prior the age of 18 is called juvenile systemic lupus erythematosus (jSLE). Previous studies have demonstrated that there are several differences in clinical manifestations and serological profiles between jSLE and adult-onset SLE (aSLE) $(3,4)$. A metaanalysis showed adverse clinical features such as malar rash, hematologic and renal involvements were more common in jSLE than in aSLE (4). Compared to aSLE, jSLE had more disease activity and a significant association with antidouble stranded (ds) DNA antibody (5).

Neutrophils, lymphocytes, and platelets play important roles in the course of various diseases. It has been demonstrated that circulating complete blood cell count $(\mathrm{CBC})$ subsets induce relative changes in systemic inflammation, which is mainly characterized by lymphopenia and neutrophilia (6). It was found that the incidence of lymphopenia was up to $82 \%$ in SLE patients, followed by leukopenia (up to $41.8 \%$ ), and neutropenia (up to $40.0 \%$ ) (7). In recent years, there has been a growing interest in the role of CBC parameters to estimate disease activity in some auto-immune diseases. Neutrophil-tolymphocyte ratio (NLR), monocyte-to-lymphocyte ratio (MLR), and platelet-to-lymphocyte ratio (PLR), as CBC parameters, have recently shown to be useful markers of inflammation in autoimmune and inflammatory disorders (8-10). Previous studies have reported that NLR and PLR were related to disease activity and organ involvement in SLE patients (11). Wu et al. found that NLR and PLR were useful inflammatory markers for assessment of SLE disease activity and an increasing of NLR was positively correlated with lupus nephritis (LN) (12). Li et al. reported NLR as a marker for SLE nephritis (13). The NLR may be used as a marker for predicting the outcome of SLE (14). Both NLR and PLR are easily available and inexpensive laboratory parameters which are convenient for clinicians to assess the disease activity.

However, data is scarce regarding the value of NLR, MLR, and PLR in jSLE patients. In this retrospective study, we analyzed the potential association between NLR, MLR, PLR, and organ involvement and disease activity in jSLE patients.

We present the following article in accordance with the STROBE reporting checklist (available at https://dx.doi. org/10.21037/apm-21-1995).

\section{Methods}

\section{Patients}

In this retrospective study cohort, 186 inpatients with onset of SLE at younger than 18 years old were enrolled from the Department of Rheumatology and Immunology of Meizhou People's Hospital between January 2010 and December 2020. All patients fulfilled at least 4 criteria of the 1997 American College of Rheumatology (ACR) revised classification criteria for SLE (15), and were newly diagnosed without having received glucocorticoid or immunosuppressant medication. Patients who co-presented with other chronic inflammatory diseases, infection, or other autoimmune diseases at the time of diagnosis were excluded. All participants were of Han Chinese ancestry. All procedures performed in this study involving human participants were in accordance with the Declaration of Helsinki (as revised in 2013). The study was approved by the Research and Ethics Review Committee of Meizhou People's Hospital (NO.: 2020-C-66). Individual consent for this retrospective analysis was waived.

\section{Clinical and serological data}

The medical records included gender, age of disease onset, age at diagnosis, clinical manifestations, laboratory data, and disease activity. The time when the first symptoms occurred was defined as the age of disease onset. According to the ACR classification criteria, malar rash, discoid rash, photosensitivity, oral ulcers, arthritis, serositis, hematological dyscrasia, lupus nephritis, and neuropsychiatric disorder were recorded as clinical manifestations at the time of diagnosis. Hematological dyscrasia included leucopenia (leucocytes less than 4,000 cells $/ \mathrm{mm}^{3}$ ), lymphopenia (lymphocytes less than 4,000 cells $/ \mathrm{mm}^{3}$ ), thrombocytopenia (platelets less than 100,000 cells $/ \mathrm{mm}^{3}$ ), and anemia. Lupus nephritis (LN) was defined as proteinuria no less than $0.5 \mathrm{~g} / 24 \mathrm{~h}$, renal biopsy, the presence of hematuria, or rising serum creatinine. Renal biopsy findings were classified according to the International Society of Nephrology/Renal Pathology Society (ISN/RPS) 2003 classification criteria (16).

The immunological data included: serum complement levels (C3 and C4), antinuclear antibodies (ANA), antidouble stranded (ds) DNA antibodies, anti-Smith (Sm) antibodies, anti-ribonucleoprotein (RNP) antibodies, antiSjögren's-syndrome-related antigen A autoantibodies (SSA)/ Ro antibodies, anti-Sjögren's-syndrome-related antigen B 
Table 1 Demographic and clinical characteristics of jSLE patients at diagnosis $(\mathrm{n}=186)$

\begin{tabular}{|c|c|}
\hline Parameter & Outcome \\
\hline \multicolumn{2}{|l|}{ Demographic characteristic } \\
\hline Female gender, n (\%) & $162(87.1)$ \\
\hline Age of onset (years), median [IQR] & $14[12-17]$ \\
\hline Age at diagnosis (years), median [IQR] & $14.5[12-17]$ \\
\hline \multicolumn{2}{|l|}{ Clinical characteristics } \\
\hline Malar rash/discoid lupus, $\mathrm{n}(\%)$ & 99 (53.2) \\
\hline Oral ulcers, n (\%) & $28(15.1)$ \\
\hline Arthritis, n (\%) & $69(37.1)$ \\
\hline LN, n (\%) & $97(52.2)$ \\
\hline Serositis, n (\%) & $42(22.6)$ \\
\hline Hematological involvement, n (\%) & $129(69.4)$ \\
\hline Neutrophils, $\times 10^{9} / \mathrm{L}$, median (IQR) & $2.85(2.1-4.2)$ \\
\hline Lymphocytes, $\times 10^{9} / \mathrm{L}$, median (IQR) & $1.3(0.9-1.9)$ \\
\hline Monocytes, $\times 10^{9} / \mathrm{L}$, median (IQR) & $0.4(0.2-0.5)$ \\
\hline Platelet, $\times 10^{9} / \mathrm{L}$, median (IQR) & $91.8(166.0-233.3)$ \\
\hline NLR, median (IQR) & $2.2(1.5-3.5)$ \\
\hline MLR, median (IQR) & $0.27(0.2-0.4)$ \\
\hline PLR, median (IQR) & $115.0(62.7-185.3)$ \\
\hline ESR, median (IQR) & $36.0(23.0-75.0)$ \\
\hline C3 level, median (IQR) & $0.36(0.22-0.67)$ \\
\hline C4 level, median (IQR) & $0.05(0.03-0.11)$ \\
\hline ANA Ab positivity, n (\%) & $186(100.0)$ \\
\hline Anti-dsDNA Ab positivity, $\mathrm{n}(\%)$ & $175(94.1)$ \\
\hline Anti-Sm Ab positivity, n (\%) & $77(41.4)$ \\
\hline Basal SLEDAI score, median (IQR) & $9.5(5.0-15.0)$ \\
\hline
\end{tabular}

jSLE, juvenile systemic lupus erythematosus; LN, lupus nephritis; NLR, neutrophil-to-lymphocyte ratio; MLR, monocyteto-lymphocyte ratio; PLR, platelet-to-lymphocyte ratio; ESR, erythrocyte sedimentation rate; $\mathrm{Ab}$, antibody; $\mathrm{C} 3$, complement component 3; C4, complement component 4; ANA, antinuclear antibodies; anti-dsDNA Ab, anti-double strained deoxyribonucleic acid antibodies; anti-Sm Ab, anti-Smith antibodies; SLEDAI, systemic lupus erythematosus disease activity index.

autoantibodies (SSB)/La antibodies, anticardiolipin (aCL) antibodies [immunoglobulin $\mathrm{G}(\mathrm{IgG})$ and immunoglobulin $M(\operatorname{IgM})$ ], and direct Coomb's test. Serum complement levels were detected by immunoturbidimetry; ANA was detected by indirect immunofluorescence using Hep-2 cells, and a titre of $\geq 1: 100$ was defined positive. Other antibodies were detected by enzyme linked immunosorbent assay (ELISA). The SLE Disease Activity Index-2000 (SLEDAI-2K) was used to assess disease activity at the time of diagnosis (17). Participants with a score $\leq 6$ were considered mild activity, those with a score of $7-12$ were considered moderate activity, and with a score $>12$ were considered highly active (18). Participants were then classified into 3 groups, as follows: mild active (SLEDAI-2K $\leq 6$ ), moderately active (SLEDAI-2K, 7-12), and highly active (SLEDAI-2K $\geq 13$ ) SLE disease.

\section{Statistical analysis}

The statistical software SPSS 22.0 (IBM Corp., Chicago, IL, USA) was used to analyze all data. Continuous variables were presented as mean \pm standard deviation (SD). Categorical variables were expressed as frequency and percentage. Mann-Whitney U test and Kruskal-Wallis test were performed for non-parametric variables between the groups. Spearman's rank correlation coefficient was used to analyze correlation between variables. A $\mathrm{P}$ value $<0.05$ was considered statistically significant.

\section{Results}

\section{Demographic data and clinical parameters}

Demographic data and clinical characteristics of 186 jSLE inpatients are shown in Table 1 . There were 162 girls $(87.1 \%)$ and 24 boys $(12.9 \%)$ in this cohort, and the ratio of female to male was $6.8: 1$. The median age of disease onset was 14 years ( $5-17$ years), and the median age of diagnosis was 14.5 years ( $5-17$ years).

The most common clinical manifestation was hematological involvement (69.4\%), followed by cutaneous rash $(53.2 \%), \mathrm{LN}(52.2 \%)$, and arthritis $(37.1 \%)$. The laboratory findings showed that ANA was positive in all participants. Anti-dsDNA antibody and anti-Sm antibody positivity were detected in 175 (94.1\%) and 77 participants (41.4\%), respectively.

\section{The comparison of NLR, MLR, and PLR in various clinical manifestations of organ involvement and disease activity in jSLE patients}

As shown in Table 2, NLR was significantly higher in 
participants with serositis as compared to those without serositis [2.72 (1.71-5.0) vs. 2.08 (1.42-3.15), $\mathrm{P}=0.038]$. Differences in MLR between jSLE patients with and without various manifestations of organ involvement did not show statistical significance. The PLR was significantly higher in participants manifesting symptoms of cutaneous rash [130.0 (92.6-235.0) vs. 112.0 (49.3-169.0), $\mathrm{P}=0.002]$, and arthritis (167.0 (106.0-243.0) vs. 106.0 (53.6-176.0), $\mathrm{P}<0.001)$, as compared to participants without such involvement. The PLR in participants with hematological involvement was significantly lower than that in those without such involvement [111.0 (53.6-191.0) vs. 138.0 (107.0-185.0), $\mathrm{P}=0.016]$. Compared to participants with negative anti-Sm antibody, PLR in participants with positive anti-Sm antibody was significantly higher [140.0 (91.6-228.0) vs. 114.0 (60.9-176.0), $\mathrm{P}=0.006]$. There was no statistically significant difference in NLR, MLR, and PLR between jSLE patients with and without positive antidsDNA antibody, low complement C3 and C4, elevated erythrocyte sedimentation rate (ESR), and SLEDAI score subgroups.

\section{Correlations of NLR, MLR and PLR with clinical parameters in jSLE patients}

We analyzed the correlations between NLR, MLR, PLR, and various clinical parameters (Table 3, Figure 1). The NLR showed positive correlations with serositis $(\mathrm{r}=0.153$, $\mathrm{P}=0.037)$, complement $\mathrm{C} 3$ and $\mathrm{C} 4[\mathrm{r}=0.152, \mathrm{P}=0.038$ and $\mathrm{r}=0.177, \mathrm{P}=0.016$, respectively]. The PLR showed positive correlations with cutaneous rash $(\mathrm{r}=0.227, \mathrm{P}=0.002)$, arthritis $(\mathrm{r}=0.290, \mathrm{P}<0.001)$, anti-Sm antibodies $(\mathrm{r}=0.20$, $\mathrm{P}=0.006)$ and $\mathrm{ESR}(\mathrm{r}=0.159, \mathrm{P}=0.03)$. Negative correlations were found between PLR and hematological involvement $(\mathrm{r}=-0.177, \mathrm{P}=0.015)$. Correlations between MLR and clinical parameters were not statistically significant.

\section{Discussion}

In the present study, our results showed that NLR was much higher in patients with serositis than in those without serositis. Besides serositis, NLR was not significantly different between participants with and without other various manifestations of organ involvement. The NLR showed positive correlations with serositis and complement C3 and C4. There were no significant differences in MLR between participants with and without various manifestations of organ involvement. Compared to those without cutaneous rash, arthritis, or hematological involvement, PLR was significantly higher in participants with cutaneous rash or arthritis, and significantly lower in those with hematological involvement. The PLR in participants with positive anti-Sm antibody was significantly higher than in those with negative anti-Sm antibody. The PLR showed positive correlations with cutaneous rash, arthritis, anti-Sm antibodies and ESR, and negative correlations with hematological involvement. However, there were no significant differences in NLR, MLR, and PLR between LN and non-LN participants. Spearman rank analysis showed that NLR, MLR, and PLR had no correlations with LN or SLEDAI-2K scores.

A previous study showed that neutrophils, lymphocytes, and platelets had complex pathophysiology which were closely related to the pathogenesis of SLE (19). Our data showed that with respect to organ involvement, significantly higher NLR was only found in participants with serositis. Previous studies have reported that NLR was not associated with serositis in jSLE or aSLE patients $(20,21)$. Suszek et al. reported that NLR was significantly higher in aSLE patients with cutaneous and/or mucosal symptoms and kidney damage (21). The NLR could reflect renal involvement in SLE patients (22). Our data showed that NLR was higher in participants with LN than those without LN, but the difference was not significant. We did not find a significant correlation between NLR and LN, which was inconsistent with a previous study (23). Some studies have found that NLR was a useful marker to assess disease activity both in jSLE and aSLE patients (11,20-22). A meta-analysis demonstrated that NLR had positive clinical value for diagnosing SLE, active SLE, and LN $(11,24)$. Ayna et al. reported a NLR cut-off value of 1.93 to differentiate SLE patients with or without nephritis (25). The NLR was positively correlated with ESR and SLEDAI scores $(22,26)$. However, the results of our study revealed no significant difference in NLR between normal and abnormal disease activity indicators, such as anti-dsDNA antibodies, ESR, complement $\mathrm{C} 3$ and $\mathrm{C} 4$, and SLEDAI score subgroups. In contrast to another study (14), NLR was positively correlated with complement $\mathrm{C} 3$ and $\mathrm{C} 4$ in our study. The NLR exhibited no correlation with SLEDAI score, which was consistent with previous reports $(21,27)$. Suszek et al. reported that MLR was significantly higher in aSLE patients manifesting symptoms of arthritis and/ or myositis (21); however, the present study did not find that MLR was significantly different between jSLE patients with and without various manifestations. The MLR had 
Table 2 A comparison of NLR, MLR, and PLR in jSLE patients in various manifestations of organ involvement and SLEDAI

\begin{tabular}{|c|c|c|c|c|c|c|}
\hline Organ involvement & NLR & $P$ value & MLR & $P$ value & PLR & $P$ value \\
\hline$+(n=99)$ & $2.30(1.48-3.48)$ & & $0.27(0.20-0.83)$ & & $130.0(92.6-235.0)$ & \\
\hline$-(n=87)$ & $2.213(1.52-3.46)$ & & $0.27(0.20-0.37)$ & & $112.0(49.3-169.0)$ & \\
\hline Arthritis & & 0.114 & & 0.792 & & $<0.001$ \\
\hline$-(n=117)$ & $2.00(1.25-3.25)$ & & $0.27(0.19-0.39)$ & & $106.0(53.6-176.0)$ & \\
\hline Hematological involvement & & 0.918 & & 0.288 & & 0.016 \\
\hline$+(n=128)$ & $2.38(1.43-3.87)$ & & $0.27(0.20-0.38)$ & & $111.0(53.6-191.0)$ & \\
\hline$-(n=58)$ & $1.55(2.10-3.17)$ & & $0.29(0.20-0.42)$ & & $138.0(107.0-185.0)$ & \\
\hline$-(n=144)$ & $2.08(1.42-3.15)$ & & $0.27(0.20-0.40)$ & & $128.0(63.3-188.0)$ & \\
\hline LN & & 0.085 & & 0.281 & & 0.967 \\
\hline$+(n=97)$ & $2.45(1.58-3.90)$ & & $0.29(0.20-0.40)$ & & $115.0(86.9-185.0)$ & \\
\hline$-(n=89)$ & $2.00(1.29-3.17)$ & & $0.25(0.17-0.38)$ & & $130.0(53.3-209.0)$ & \\
\hline Anti-dsDNA Ab & & 0.849 & & 0.264 & & 0.268 \\
\hline$+(n=175)$ & $2.25(1.52-3.43)$ & & $0.27(0.20-0.40)$ & & $124.0(76.4-190.0)$ & \\
\hline$-(n=11)$ & $1.69(1.08-5.18)$ & & $0.24(0.17-0.30)$ & & $106.0(34.9-728.0)$ & \\
\hline Anti-Sm Ab & & 0.598 & & 0.645 & & 0.006 \\
\hline Low C3 & & 0.708 & & 0.242 & & 0.673 \\
\hline$+(n=159)$ & $2.15(1.47-3.51)$ & & $0.27(0.20-0.38)$ & & $118.0(71.8-186.0)$ & \\
\hline$-(n=27)$ & $2.45(1.55-3.50)$ & & $0.35(0.22-0.42)$ & & $129.0(82.1-222.0)$ & \\
\hline Low C4 & & 0.304 & & 0.814 & & 0.202 \\
\hline$+(n=133)$ & $2.15(1.38-3.25)$ & & $0.27(0.20-0.40)$ & & $115.0(75.6-180.0)$ & \\
\hline$-(n=53)$ & $2.38(1.67-3.71)$ & & $0.33(0.20-0.40)$ & & $140.0(64.4-243.0)$ & \\
\hline SLEDAI & & 0.067 & & 0.378 & & 0.645 \\
\hline$\leq 6(n=65)$ & $2.67(1.35-3.79)$ & & $0.27(0.20-0.40)$ & & $123.85(36.88-187.96)$ & \\
\hline $7-12(n=57)$ & $1.94(1.32-2.80)$ & & $0.25(0.18-0.34)$ & & 128.50 (73.29-191.83) & \\
\hline$\geq 13(n=64)$ & $2.44(1.58-4.20)$ & & $0.30(0.20-0.40)$ & & $120.97(88.96-190.08)$ & \\
\hline
\end{tabular}

NLR, neutrophil-to-lymphocyte ratio; MLR, monocyte-to-lymphocyte ratio; PLR, platelet-to-lymphocyte ratio; jSLE, juvenile systemic lupus erythematosus; SLEDAI, systemic lupus erythematosus disease activity index; LN, lupus nephritis; anti-dsDNA Ab, anti-double strained deoxyribonucleic acid antibodies; anti-Sm Ab, anti-Smith antibodies; ESR, erythrocyte sedimentation rate; C3, complement component 3; C4, complement component 4. 
Table 3 Correlations of clinical parameters with NLR, MLR, and PLR in jSLE patients

\begin{tabular}{|c|c|c|c|c|c|c|}
\hline & \multicolumn{2}{|c|}{ NLR } & \multicolumn{2}{|c|}{ MLR } & \multicolumn{2}{|c|}{ PLR } \\
\hline Cutaneous rash & 0.004 & 0.962 & 0.016 & 0.828 & 0.227 & $0.002^{* *}$ \\
\hline Arthritis & 0.116 & 0.115 & 0.019 & 0.793 & 0.290 & $<0.001^{\star \star}$ \\
\hline Hematological involvement & 0.008 & 0.918 & -0.078 & 0.289 & -0.177 & $0.015^{\star}$ \\
\hline LN & 0.127 & 0.085 & 0.079 & 0.282 & 0.003 & 0.968 \\
\hline Anti-dsDNA Ab & 0.014 & 0.849 & 0.082 & 0.265 & 0.082 & 0.269 \\
\hline Anti-Sm Ab & 0.039 & 0.60 & 0.034 & 0.646 & 0.20 & $0.006^{\star *}$ \\
\hline ESR & 0.017 & 0.819 & 0.044 & 0.554 & 0.159 & $0.030^{*}$ \\
\hline SLEDAI & 0.095 & 0.197 & 0.040 & 0.586 & 0.114 & 0.122 \\
\hline
\end{tabular}

*, $\mathrm{P}<0.05 ;{ }^{* *}, \mathrm{P}<0.01$. NLR, neutrophil-to-lymphocyte ratio; MLR, monocyte-to-lymphocyte ratio; PLR, platelet-to-lymphocyte ratio; jSLE, juvenile systemic lupus erythematosus; LN, lupus nephritis; anti-dsDNA Ab, anti-double strained deoxyribonucleic acid antibodies; anti$\mathrm{Sm}$ Ab, anti-Smith antibodies; ESR, erythrocyte sedimentation rate; C3, complement component 3; C4, complement component 4; SLEDAI, systemic lupus erythematosus disease activity index.

no correlation with SLEDAI score in the present study, which was consistent with a previous report (27). Liu et al. found that MLR may be useful biomarkers in predicting LN (27), however we did not found correlation between MLR and LN.

Previous studies have reported that PLR was significantly different between SLE patients and healthy controls $(11,12,23)$. The results of various studies were inconsistent regarding whether PLN was related to clinical manifestations and disease activity. Peirovy et al. did not find a significant difference in PLR between aSLE patients with and without cutaneous rash, arthritis, and serositis (23). The PLR was significantly higher in SLE patients exhibiting hematological disorders compared to those without such involvement, and it was not significantly different between patients with and without cutaneous, arthritis, or anti-dsDNA antibody (21). The present study showed that PLR was significantly different between participants with and without cutaneous rash, arthritis, hematological involvement, and anti-Sm antibodies. The PLR was not significantly different between participants with and without positive anti-dsDNA antibodies, which was consistent with a previous report (21). Consistent with previous studies $(22,23)$, we also found that PLR was not significantly different between participants with and without LN. In contrast to our finding, another study reported that PLR was significantly higher in SLE patients exhibiting LN (21). The PLR was positively correlated with SLEDAI and may be useful marker to evaluate SLE disease activity (11). The same findings were reported in both aSLE $(22,23)$ and jSLE patients $(20)$. While in the present study, we did not find that PLR was correlated with SLEDAI, which was consistent with a previous study (27). These inconsistent findings in PLR may be attributable to factors such as sample types and size, genetic background, and analytical methods.

The NLR and PLR had been widely studied in SLE, but their positive clinical value for diagnosing SLE were remain controversial. Previous study found that NLR had positive clinical value for diagnosing SLE (24). Several studies did not found NLR and PLR had positive clinical value for diagnosing SLE $(12,14,21)$. Previous studies found increased levels of NLR and PLR in SLE patients as compared to healthy controls $(11,22)$. The levels of NLR and PLR were also elevated in malignancies (28), infectious diseases (29) and other autoimmune diseases such as primary Sjögren's syndrome (PSS) (30), psoriasis (31) and ulcerative colitis (32). The anti-ds DNA antibody and anti-Sm antibody are remainly serological markers in the new classification criteria for SLE (33). It demonstrated that 

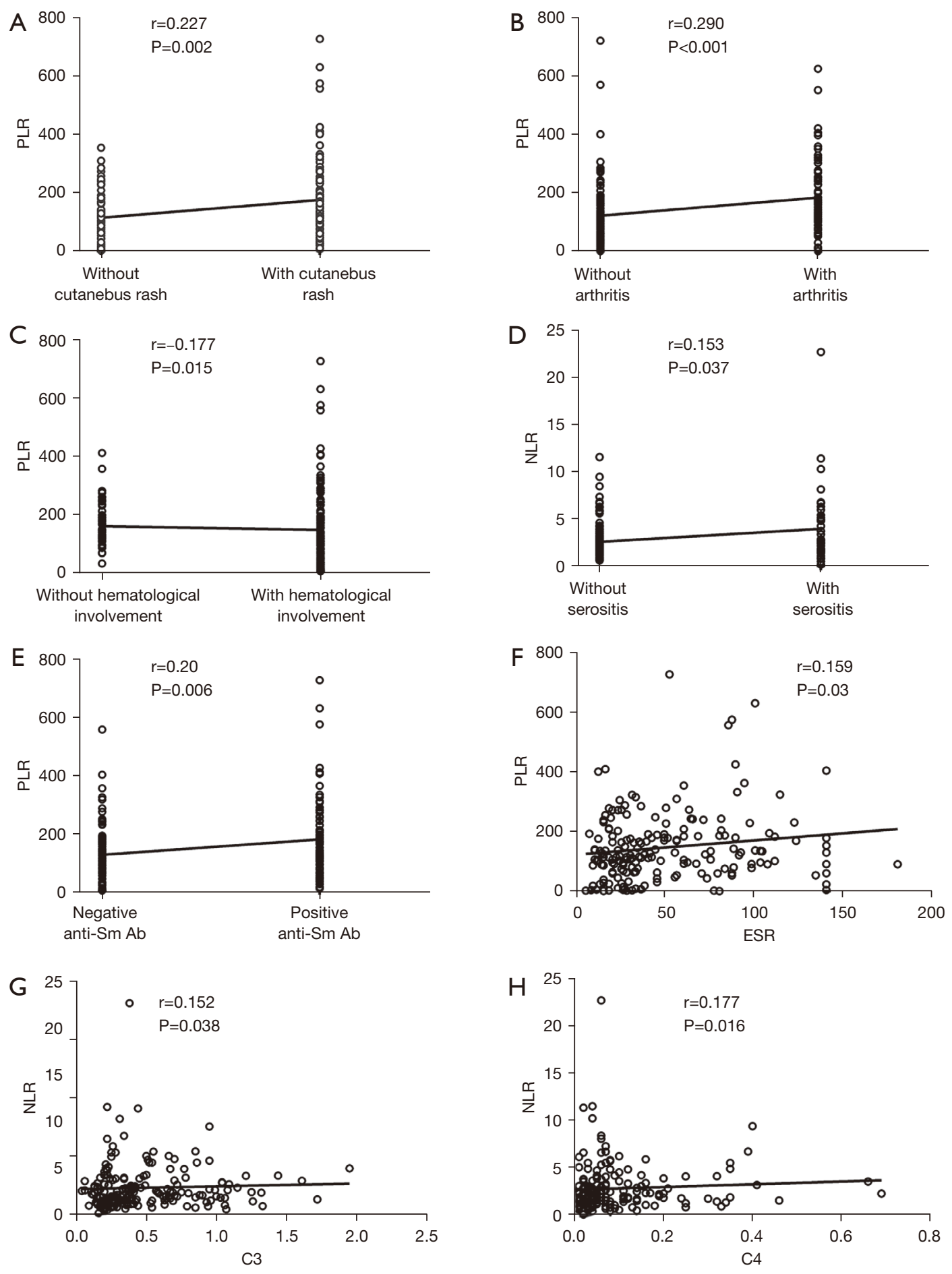

Figure 1 The correlations of NLR, MLR and PLR with clinical parameters in jSLE patients. (A) Correlation between cutaneous rash and PLR; (B) correlation between arthritis and PLR; (C) correlation between hematological involvement and PLR; (D) correlation between serositis and NLR; (E) correlation between anti-Sm Ab and PLR; (F) correlation between ESR and PLR; (G) correlation between C3 and NLR; (H) correlation between C4 and NLR. PLR, platelet-to-lymphocyte ratio; NLR, neutrophil-to-lymphocyte ratio; anti-Sm Ab, antiSmith antibodies; ESR, erythrocyte sedimentation rate; C3, complement component 3; C4, complement component 4; MLR, monocyte-tolymphocyte ratio; jSLE, juvenile systemic lupus erythematosus. 
NLR and PLR were not the specific markers for the diagnosis of SLE.

However, there were some limitations to this study. Firstly, it was a retrospective design study. Secondly, the sample size was relatively small. Finally, this study was conducted in a single-center. Further studies, preferably multicenter studies, should be performed to describe the value of peripheral blood cell ratios in jSLE.

In conclusion, the present study revealed that NLR and PLR had correlations with serological indicators, and may predict organ involvement in jSLE, particularly cutaneous, arthritis, serositis, and hematological involvement.

\section{Acknowledgments}

Funding: This study was supported by Scientific Research Talent Cultivation Fund of Meizhou People's Hospital (PYC2020009).

\section{Footnote}

Reporting Checklist: The authors have completed the STROBE reporting checklist. Available at https://dx.doi. org/10.21037/apm-21-1995

Data Sharing Statement: Available at https://dx.doi. org/10.21037/apm-21-1995

Conflicts of Interest: All authors have completed the ICMJE uniform disclosure form (available at https://dx.doi. org/10.21037/apm-21-1995). The authors have no conflicts of interest to declare.

Ethical Statement: The authors are accountable for all aspects of the work in ensuring that questions related to the accuracy or integrity of any part of the work are appropriately investigated and resolved. All procedures performed in this study involving human participants were in accordance with the Declaration of Helsinki (as revised in 2013). The study was approved by the Research and Ethics Review Committee of Meizhou People's Hospital (NO.: 2020-C-66). Individual consent for this retrospective analysis was waived.

Open Access Statement: This is an Open Access article distributed in accordance with the Creative Commons Attribution-NonCommercial-NoDerivs 4.0 International License (CC BY-NC-ND 4.0), which permits the non- commercial replication and distribution of the article with the strict proviso that no changes or edits are made and the original work is properly cited (including links to both the formal publication through the relevant DOI and the license). See: https://creativecommons.org/licenses/by-nc-nd/4.0/.

\section{References}

1. Rahman A, Isenberg DA. Systemic lupus erythematosus. N Engl J Med 2008;358:929-39.

2. Sassi RH, Hendler JV, Piccoli GF, et al. Age of onset influences on clinical and laboratory profile of patients with systemic lupus erythematosus. Clin Rheumatol 2017;36:89-95.

3. Artim-Esen B, Şahin S, Çene E, et al. Comparison of Disease Characteristics, Organ Damage, and Survival in Patients with Juvenile-onset and Adult-onset Systemic Lupus Erythematosus in a Combined Cohort from 2 Tertiary Centers in Turkey. J Rheumatol 2017;44:619-25.

4. Bundhun PK, Kumari A, Huang F. Differences in clinical features observed between childhood-onset versus adultonset systemic lupus erythematosus: A systematic review and meta-analysis. Medicine (Baltimore) 2017;96:e8086.

5. Chen C, Geng L, Xu X, et al. Comparative proteomics analysis of plasma protein in patients with neuropsychiatric systemic lupus erythematosus. Ann Transl Med 2020;8:579.

6. Li Z, Xiao Y, Zhang L. Application of procalcitonin, white blood cell count and neutrophil-to-lymphocyte ratio in the diagnosis of systemic lupus erythematosus with a bacterial infection. Ann Palliat Med 2020;9:3870-6.

7. Carli L, Tani C, Vagnani S, et al. Leukopenia, lymphopenia, and neutropenia in systemic lupus erythematosus: Prevalence and clinical impact--A systematic literature review. Semin Arthritis Rheum 2015;45:190-4.

8. Hao X, Li D, Wu D, et al. The Relationship between Hematological Indices and Autoimmune Rheumatic Diseases (ARDs), a Meta-Analysis. Sci Rep 2017;7:10833.

9. Gasparyan AY, Ayvazyan L, Mukanova U, et al. The Platelet-to-Lymphocyte Ratio as an Inflammatory Marker in Rheumatic Diseases. Ann Lab Med 2019;39:345-57.

10. Yang Z, Zhang Z, Lin F, et al. Comparisons of neutrophil-, monocyte-, eosinophil-, and basophil- lymphocyte ratios among various systemic autoimmune rheumatic diseases. APMIS 2017;125:863-71.

11. Ma L, Zeng A, Chen B, et al. Neutrophil to lymphocyte ratio and platelet to lymphocyte ratio in patients with systemic lupus erythematosus and their correlation 
with activity: A meta-analysis. Int Immunopharmacol 2019;76:105949.

12. Wu Y, Chen Y, Yang X, et al. Neutrophil-to-lymphocyte ratio (NLR) and platelet-to-lymphocyte ratio (PLR) were associated with disease activity in patients with systemic lupus erythematosus. Int Immunopharmacol 2016;36:94-9.

13. Li L, Xia Y, Chen C, et al. Neutrophil-lymphocyte ratio in systemic lupus erythematosus disease: a retrospective study. Int J Clin Exp Med 2015;8:11026-31.

14. Yu H, Jiang L, Yao L, et al. Predictive value of the neutrophil-to-lymphocyte ratio and hemoglobin insystemic lupus erythematosus. Exp Ther Med 2018;16:1547-53.

15. Hochberg MC. Updating the American College of Rheumatology revised criteria for the classification of systemic lupus erythematosus. Arthritis Rheum 1997;40:1725.

16. Weening JJ, D'Agati VD, Schwartz MM, et al. The classification of glomerulonephritis in systemic lupus erythematosus revisited. Kidney Int 2004;65:521-30.

17. Gladman DD, Ibañez D, Urowitz MB. Systemic lupus erythematosus disease activity index 2000. J Rheumatol 2002;29:288-91.

18. Chinese Rheumatology Association; National Clinical Research Center for Dermatologic and Immunologic Diseases; Chinese Systemic Lupus Erythematosus Treatment and Research Group. 2020 Chinese guidelines for the diagnosis and treatment of systemic lupus erythematosus. Zhonghua Nei Ke Za Zhi 2020;59:172-85.

19. Linge P, Fortin PR, Lood C, et al. The non-haemostatic role of platelets in systemic lupus erythematosus. Nat Rev Rheumatol 2018;14:195-213.

20. Luo KL, Yang YH, Lin YT, et al. Differential parameters between activity flare and acute infection in pediatric patients with systemic lupus erythematosus. Sci Rep 2020;10:19913.

21. Suszek D, Górak A, Majdan M. Differential approach to peripheral blood cell ratios in patients with systemic lupus erythematosus and various manifestations. Rheumatol Int 2020;40:1625-9.

22. Soliman WM, Sherif NM, Ghanima IM, et al. Neutrophil to lymphocyte and platelet to lymphocyte ratios in systemic lupus erythematosus: Relation with disease activity and lupus nephritis. Reumatol Clin (Engl Ed) 2020;16:255-61.

23. Peirovy A, Malek Mahdavi A, Khabbazi A, et al. Clinical Usefulness of Hematologic Indices as Predictive Parameters for Systemic Lupus Erythematosus. Lab Med 2020;51:519-28.

24. Wang L, Wang C, Jia X, et al. Relationship between
Neutrophil-to-Lymphocyte Ratio and Systemic Lupus Erythematosus: A Meta-analysis. Clinics (Sao Paulo) 2020;75:e1450.

25. Ayna AB, Ermurat S, Coşkun BN, et al. Neutrophil to Lymphocyte Ratio and Mean Platelet Volume as Inflammatory Indicators in Systemic Lupus Erythematosus Nephritis. Arch Rheumatol 2017;32:21-5.

26. Qin B, Ma N, Tang Q, et al. Neutrophil to lymphocyte ratio (NLR) and platelet to lymphocyte ratio (PLR) were useful markers in assessment of inflammatory response and disease activity in SLE patients. Mod Rheumatol 2016;26:372-6.

27. Liu P, Li P, Peng Z, et al. Predictive value of the neutrophil-to-lymphocyte ratio, monocyte-to-lymphocyte ratio, platelet-to-neutrophil ratio, and neutrophil-tomonocyte ratio in lupus nephritis. Lupus 2020;29:1031-9.

28. Koh CH, Bhoo-Pathy N, Ng KL, et al. Utility of pretreatment neutrophil-lymphocyte ratio and plateletlymphocyte ratio as prognostic factors in breast cancer. $\mathrm{Br}$ J Cancer 2015;113:150-8.

29. de Jager CP, van Wijk PT, Mathoera RB, et al. Lymphocytopenia and neutrophil-lymphocyte count ratio predict bacteremia better than conventional infection markers in an emergency care unit. Crit Care 2010;14:R192.

30. Hu ZD, Sun Y, Guo J, et al. Red blood cell distribution width and neutrophil/lymphocyte ratio are positively correlated with disease activity in primary Sjögren's syndrome. Clin Biochem 2014;47:287-90.

31. Sen BB, Rifaioglu EN, Ekiz O, et al. Neutrophil to lymphocyte ratio as a measure of systemic inflammation in psoriasis. Cutan Ocul Toxicol 2014;33:223-7.

32. Akpinar MY, Ozin YO, Kaplan M, et al. Platelet-tolymphocyte Ratio and Neutrophil-to-lymphocyte Ratio Predict Mucosal Disease Severity in Ulcerative Colitis. J Med Biochem 2018;37:155-62.

33. Aringer M, Costenbader K, Daikh D, et al. 2019 European League Against Rheumatism/American College of Rheumatology classification criteria for systemic lupus erythematosus. Ann Rheum Dis 2019;78:1151-9.

Cite this article as: Li W, Liu S, Chen C, Han Y. Neutrophilto-lymphocyte ratios and platelet-to-lymphocyte ratios in juvenile systemic lupus erythematosus: correlation with disease manifestations. Ann Palliat Med 2021;10(9):9406-9414. doi: 10.21037/apm-21-1995 